\title{
Parâmetros de crescimento e suas correlações em idades entre 60 a 240 dias na carpa hungara (Cyprinus carpio)
}

\section{Growth parameters and their correlations from ages 60 to 240 days in the carp (Cyprinus carpio)}

\author{
Marco Antonio da Rocha ${ }^{1 *}$; Edson Luis de Azambuja Ribeiro ${ }^{1}$; \\ Ivone Yurika Mizubuti ${ }^{1}$; Leandro das Dores Ferreira da Silva ${ }^{1}$
}

\section{Resumo}

O presente trabalho teve como objetivo a determinação de parâmetros de crescimento e suas correlações na carpa em idades entre 60 a 240 dias. No experimento foram utilizados 65 peixes, sendo 31 machos e 34 fêmeas oriundos da mesma desova e criados nas mesmas condições ambientais. A diferença entre sexos não foi encontrada $(\mathrm{P}>0,05)$ para todas as características em nenhuma das idades mencionadas. O crescimento isométrico para ambos os sexos foi para as relações: Peso x Altura e Peso x Comprimento da cabeça. As correlações entre as características foram de moderado a alto $(\mathrm{P}<0,01)$, à exceção do comprimento corporal aos 60 dias com comprimento corporal, altura corporal e comprimento da cabeça na idade de 240 dias, que foram baixos $(\mathrm{P}<0,05)$.

Palavras chave: Peixes; Crescimento; Correlações.

\begin{abstract}
This work had as objective to determine growth parameters and their correlations from ages 60 to 240 days in the carp. The experiment used 65 fishes, 31 males and 34 females, from the same hatch and raised in the same environmental conditions. There were no differences $(\mathrm{P}>0.05)$ between sexes for all studied traits. The relationship between body weight $\mathrm{x}$ height and body weight $\mathrm{x}$ head length presented isometric growth for both sexes. Moderate to high correlations $(\mathrm{P}<0.01)$ were found between traits, the exceptions were for body length at 60 days with body length, body heigth and head at 240 days, which presented small correlations $(\mathrm{P}<0.05)$.
\end{abstract}

Key-word: Fish; Gowth; Correlations.

\section{Introdução}

A piscicultura é uma atividade em grande expansão, principalmente no Brasil onde os recursos hídricos são extraordinários e representados por 7048 $\mathrm{km}$ de costa marítima e $55.457 \mathrm{~km}^{2}$ de águas interiores. Entretanto, o país não é destaque entre os maiores produtores de pescado.
Para o incremento desta atividade no país alguns fatores básicos devem ser levados em consideração, tais como: manejo correto de bacias hidrográficas, preservação dos estoques selvagens, incentivo às pesquisas básicas e aplicadas e difusão destes resultados, conforme Rocha (1995).

O crescimento dos peixes é um fator importante

\footnotetext{
${ }^{1}$ Professores associados do Departamento de Zootecnia da Universidade Estadual de Londrina, Caixa Postal 6001, CEP 86051990, Londrina, Paraná, Brasil.

* Autor para correspondência.
} 
e que determina o potencial das várias espécies para a exploração econômica.

A relação entre as características de mesma unidade de medida é considerada isométrica quando o coeficiente de regressão é igual a 1,0. Quando os valores são estatisticamente maiores ou menores que 1,0 , indicam uma relação alométrica, isto é, o crescimento diferenciado de uma característica em relação à outra. Nas relações entre características de unidade de medidas diferentes, o crescimento é considerado isométrico quando $b=3,0$. Os valores estatisticamente maiores ou menores que 3,0 são classificados como alométrico (RICKER, 1979).

A correlação ou o grau de associação entre as características é um parâmetro muito importante na avaliação de uma característica pela outra, principalmente quando estas são avaliadas em idades diferentes.

Muitos pesquisadores, tais como, Tave e Smitherman, (1980); Busack e Gall, (1983); El Ibiary et al (1978); Bailei e Loudenslager, (1986); Reagan, Pardue e Eisen; (1976); Refstie e Steine, (1978); Rocha, (1995), trabalhando com diversas espécies de peixes, tem evidenciado uma associação moderada a alta e positiva entre características avaliadas em idades diferentes, o que indica que nos peixes com maiores valores em uma determinada idade provavelmente o serão também em outras idades.

O objetivo deste trabalho foi o de determinar em uma amostra de carpa hungara (Cyprinus carpio), os parâmetros de crescimento, considerando os sexos, em animais criados nas mesmas condições ambientais.

\section{Material e Métodos}

O experimento foi realizado no período de março a outubro de 1995, na Estação de Piscicultura do Centro de Ciências Biológicas na Universidade Estadual de Londrina- Paraná.

A amostra foi constituída de 65 carpas (Cyprinus cárpio), sendo 34 fêmeas e 31 machos oriundos da mesma desova, criados em um viveiro de $100 \mathrm{~m} 2$, recebendo ração para crescimento com $30 \%$ de proteína bruta e $2800 \mathrm{kcal}$ de energia, e identificados com marcação à ferro incandescente, em idades entre 60 a 240 dias. Foi determinado neste viveiro, durante todo o período experimental, algumas características da água como: temperatura, $\mathrm{pH}$, condutividade elétrica e oxigênio dissolvido.

As características avaliadas nas idades de 60, 120, 180 e 240 dias, foram:

1 - Peso (P) - Determinado em balança de precisão, expresso em gramas.

2 - Comprimento total (C) - Esta característica foi determinada com o auxilio de um paquímetro, tendo como base a ponte do focinho até a extremidade do ramo da nadadeira caudal, e expresso em centímetros.

3 - Altura (A) - Foi obtido com paquímetro tendo como base o início da nadadeira dorsal até a base do ventre, e expresso em centímetros.

4 - Comprimento da cabeça (Cab) - Foi medido com paquímetro tendo como base a ponta do focinho até a borda posterior do opérculo, expresso em centímetros.

As relações entre peso e altura; altura e comprimento total; peso e comprimento total; comprimento da cabeça e comprimento total; peso corporal e comprimento da cabeça; comprimento da cabeça e altura foram determinados para verificar se estas relações são isométricas e alométricas.

Para o cálculo, as variáveis foram transformadas para logaritmo natural. Em seguida, foram determinados o A (intercepto) e o B (coeficiente de regressão). O intercepto calculado através da transformação não representa a constante da relação, sendo necessário a determinação do antilogarítmo natural para fornecer o valor do intercepto. O teste de isometria e alometria ao nível de significância de $5 \%$ foi determinado através do teste " $\mathrm{t}$ ", conforme Sokal e Rohlf (1980). 
Os dados foram analisados, conforme o modelo matemático:

$$
Y_{i j}=\mu+S_{i}+E_{i j}
$$

Sendo que:

$\mathrm{Y}_{\mathrm{ij}}=$ Peso, comprimento total, altura e comprimento da cabeça, de animais de sexo macho ou fêmea, nas idades de 60, 120, 180 e 240 dias,

$\mu=$ Média geral,

$\mathrm{S}_{\mathrm{i}}=$ Efeito do sexo do animal (1-macho e 2-fêmea),

$\mathrm{E}_{\mathrm{ij}}=$ Efeito do erro experimental.

As correlações entre as características nas várias idades, 60 até 240 dias, e a significância estatística utilizando o teste "t" ao nível de 5\% e 1\%, foi feito através do programa SAEG conforme recomendação de Euclydes (1983).

\section{Resultados e discussão}

Durante o período experimental, a qualidade da água apresentou os seguintes resultados conforme os variáveis analisados e que constam na tabela 1 .

Tabela 1 - Número de avaliações $(\mathrm{N})$, médias $(\mathrm{X})$, desvio padrão (s) e coeficiente de variação $(\mathrm{CV})$ para as características: temperatura, $\mathrm{pH}$, condutividade elétrica e oxigênio dissolvido, avaliados no viveiro de criação.

\begin{tabular}{lcccc}
\hline Características & $\mathbf{N}$ & $\overline{\mathbf{X}}$ & $\mathbf{S}$ & $\mathbf{C V}(\boldsymbol{\%})$ \\
& & & & \\
\hline Temperatura $\left({ }^{0} \mathrm{C}\right)$ & 12 & 22,42 & 2,56 & 10,79 \\
PH & 12 & 6,82 & 0,50 & 7,26 \\
Condutividade elétrica $\left(\mu \mathrm{s} \mathrm{cm}^{-1}\right)$ & 12 & 59,08 & 6,73 & 11,38 \\
Oxigênio dissolvido $\left(\mathrm{mg} \mathrm{l}^{-1}\right)$ & 12 & 6,75 & 0,62 & 9,20 \\
\hline
\end{tabular}

Os valores da Tabela 1 indicam que estão dentro do limite apresentado por Padua (1994). Desta forma, as condições ambientais representadas pelas variáveis avaliadas na água do viveiro de criação foram compatíveis durante todo o experimento, com valores recomendados para a manutenção de organismos aquáticos de água doce.

Na Tabela 2 são apresentados as médias e erros padrão para as características: peso, comprimento total, altura e comprimento da cabeça, conforme os sexos.

Tabela 2 - Médias ajustadas $(\overline{\mathrm{X}})$ e erros padrão (EP) para as características: Peso corporal $(\mathrm{P})$; comprimento corporal (C); altura corporal (A) e comprimento de cabeça (CAB) em idades entre 60 e 240 dias, para machos e fêmeas da carpa.

\begin{tabular}{|c|c|c|c|c|}
\hline \multirow[t]{2}{*}{ Características } & \multicolumn{2}{|c|}{ Machos } & \multicolumn{2}{|l|}{ Fêmeas } \\
\hline & $\overline{\mathbf{X}} \pm$ & EP & $\overline{\mathbf{X}} \pm$ & $\mathbf{E P}$ \\
\hline $\mathrm{P}_{60}(\mathrm{~g})$ & 23,99 & $\pm 2,06$ & 29,25 & $\pm 3,09$ \\
\hline $\mathrm{C}_{60}(\mathrm{~cm})$ & 10,50 & $\pm 0,27$ & 10,98 & $\pm 0,31$ \\
\hline $\mathrm{A}_{60}(\mathrm{~cm})$ & 3,61 & $\pm 0,11$ & 3,86 & $\pm 0,14$ \\
\hline $\mathrm{CAB}_{60}(\mathrm{~cm})$ & 2,89 & $\pm 0,09$ & 3,05 & $\pm 0,11$ \\
\hline $\mathrm{P}_{120}(\mathrm{~g})$ & 64,72 & $\pm 5,19$ & 68,31 & $\pm 5,53$ \\
\hline $\mathrm{C}_{120}(\mathrm{~cm})$ & 14,97 & $\pm 0,34$ & 15,22 & $\pm 0,35$ \\
\hline $\mathrm{A}_{120}(\mathrm{~cm})$ & 4,91 & $\pm 0,13$ & 4,99 & $\pm 0,15$ \\
\hline $\mathrm{CAB}_{120}(\mathrm{~cm})$ & 3,85 & $\pm 0,10$ & 4,00 & $\pm 0,10$ \\
\hline$P_{180}(g)$ & 81,53 & $\pm 6,13$ & 97,65 & $\pm 7,15$ \\
\hline $\mathrm{C}_{180}(\mathrm{~cm})$ & 16,41 & $\pm 0,36$ & 17,11 & $\pm 0,42$ \\
\hline $\mathrm{A}_{180}(\mathrm{~cm})$ & 5,22 & $\pm 0,18$ & 5,50 & $\pm 0,17$ \\
\hline $\mathrm{CAB}_{180}(\mathrm{~cm})$ & 4,20 & $\pm 0,11$ & 4,40 & $\pm 0,18$ \\
\hline $\mathrm{P}_{240}(\mathrm{~g})$ & 144,45 & $\pm 7,89$ & 155,02 & $\pm 9,59$ \\
\hline $\mathrm{C}_{240}(\mathrm{~cm})$ & 19,64 & $\pm 0,42$ & 20,33 & $\pm 0,44$ \\
\hline $\mathrm{A}_{240}(\mathrm{~cm})$ & 6,28 & $\pm 0,13$ & 6,42 & $\pm 0,15$ \\
\hline $\mathrm{CAB}_{240}(\mathrm{~cm})$ & 4,99 & $\pm 0,11$ & 5,23 & $\pm 0,10$ \\
\hline
\end{tabular}

Em todas as idades, os valores médios das características foram maiores nas fêmeas do que nos machos. No entanto, estas diferenças não foram significativas $(\mathrm{P}>0,05)$.

A dispersão foi mais evidente na característica peso corporal nas várias idades, concordando com os resultados apresentados por Gjedrem (1983). Por outro lado, a dispersão observada para as características: comprimento total, altura e comprimento da 
cabeça, nas várias idades, apresentaram uma baixa dispersão, o que mostra a uniformidade dos valores obtidos, e coincidindo com os resultados encontrados por Gjedrem (1983).

Na Tabela 3 são apresentados as relações entre as características de crescimento: peso, comprimento total, altura e comprimento da cabeça, conforme os sexos.

Tabela 3 - Valores do intercepto (lna), Coeficiente de regressão (b), Coeficiente de determinação $\left(r^{2}\right)$ e tipo de crescimento (Cresc.) para as relações entre as características: Peso (P); Comprimento total (C); Altura (A) e comprimento da cabeça (CAB), conforme os sexos (machos (1) e fêmeas (2)), na carpa.

\begin{tabular}{llllll}
\hline Relação & Sexos & Ina & b & $\mathbf{R}^{2}$ & Crescimento \\
& & & & & \\
\hline P X A & 1 & 0,5357 & 2,99 & 0,93 & Iso \\
& 2 & 0,5353 & 2,98 & 0,95 & Iso \\
& Total & 0,5380 & 2,98 & 0,94 & Iso \\
& & & & & \\
\hline A X C & 1 & 0,4217 & 0,90 & 0,91 & Alo \\
& 2 & 0,4478 & 0,88 & 0,87 & Alo \\
& Total & 0,4347 & 0,89 & 0,89 & Alo \\
& & & & & \\
\hline P X C & 1 & 0,023988 & 2,90 & 0,97 & Alo \\
& 2 & 0,029974 & 2,82 & 0,94 & Alo \\
& Total & 0,027190 & 2,86 & 0,94 & Alo \\
& & & & & \\
\hline CAB X C & 1 & 0,3469 & 0,89 & 0,93 & Alo \\
& 2 & 0,3321 & 0,88 & 0,87 & Alo \\
& Total & 0,3532 & 0,89 & 0,90 & Alo \\
& & & & & \\
\hline P X CAB & 1 & 0,9571 & 3,07 & 0,93 & Iso \\
& 2 & 1,0978 & 2,96 & 0,92 & Iso \\
& Total & 1,0360 & 3,00 & 0,92 & Iso \\
& & & & & \\
\hline CAB X A & 1 & 0,9018 & 0,92 & 0,89 & Alo \\
& 2 & 0,8671 & 0,95 & 0,91 & Alo \\
& Total & 0,8822 & 0,94 & 0,90 & Alo \\
& & & & & \\
\hline
\end{tabular}

Alométrico (Alo) ( $\mathrm{P}<0,05)$; Isométrico (Iso) $(\mathrm{P}>0,05)$, pelo teste " $\mathrm{t}$ ".

A relação entre peso e altura apresentou um crescimento isométrico $(\mathrm{P}>0,05)$ com o coeficiente de regressão (b) igual a 2,98. O crescimento separado por sexo foi isométrico $(\mathrm{P}>0,05)$ para ambos os sexos, machos $(b=2,99)$ e fêmeas $(b=2,98)$. Estes resultados indicam que o crescimento foi similar para o peso e para a altura, o que discorda do resultado obtido por Rocha (1995) no curimbatá (Prochilodus lineatus).

Para a relação entre altura e o comprimento, o crescimento observado foi alométrico negativo $(\mathrm{P}<0,05)$ com $\mathrm{b}=0,89$. Em relação aos sexos, o tipo de crescimento foi o alométrico negativo $(\mathrm{P}<0,05)$ para machos $(b=0,90)$ e fêmeas $(b=0,88)$. Isto indica que a altura teve crescimento proporcional mais acentuado do que o comprimento total, discordando do resultado obtido por Rocha (1995) que determinou um crescimento isométrico no curimbatá.

A relação entre peso e comprimento total apresentou crescimento alométrico negativo $(\mathrm{P}<0,05)$ com $b=2,86$. Este valor está dentro da faixa de valores citados em Le Cren (1951) e Royce (1972) e próximo aos valores encontrados por Gunnes e Gjedrem (1981); Gjerde e Schaffer (1989) na truta arco iris (Salmo gairdneri); Refstie e Steine (1978) no salmão e Rocha (1995) no curimbatá. Este mesmo tipo de crescimento foi observado entre os machos $(b=2,90)$ e entre as fêmeas $(b=2,82)$. O resultado indica que o peso teve um crescimento maior que o comprimento total, o que concorda com o determinado por Rocha (1995).

A relação entre o comprimento da cabeça e o comprimento total, apresentou também crescimento alométrico negativo $(P<0,05)$ com $b=0,89$. Na separação por sexos, o crescimento observado foi alométrico negativo $(\mathrm{P}<0,05)$, tanto em machos $(b=0,89)$ como em fêmeas $(b=0,88)$, concordando com o valor apresentado por Rocha (1995).

Para o peso e comprimento da cabeça o crescimento foi isométrico $(\mathrm{P}>0,05)$ com $b=3,00$, e igualmente em relação aos sexos, ou seja, isométrico ( $P>0,05$ ) com $b=3,07$ para os machos e $b=2,96$ para as fêmeas.

A relação entre comprimento da cabeça e altura foi alométrica negativa $(P<0,05)$ com $b=0,94$. O mesmo ocorreu em relação aos sexos, machos $(b=0,92)$ e fêmeas $(b=0,95)$. 
Na Tabela 4 são apresentados as correlações fenotípicas entre as características: peso, comprimen- to total, altura e o comprimento da cabeça, nas diversas idades.

Tabela 4 - Correlações entre as características: Peso (P); Comprimento (C); Altura (A) e o Comprimento da cabeça (CAB) nas idades de 60, 120, 180 e 240 dias na carpa.

\begin{tabular}{|c|c|c|c|c|c|c|c|c|c|c|c|c|c|c|c|}
\hline & $\mathrm{C}_{60}$ & $\mathrm{~A}_{60}$ & $\mathrm{CAB}_{60}$ & $\mathrm{P}_{120}$ & $\mathrm{C}_{120}$ & $\mathrm{~A}_{120}$ & $\mathrm{CAB}_{120}$ & $\mathrm{P}_{180}$ & $\mathrm{C}_{180}$ & $\mathrm{~A}_{180}$ & $\mathrm{CAB}_{180}$ & $\mathrm{P}_{240}$ & $\mathrm{C}_{240}$ & $\mathrm{~A}_{240}$ & $\mathrm{CAB}_{240}$ \\
\hline $\mathrm{P}_{60}$ & 0,82 & 0,98 & 0,89 & 0,83 & 0,79 & 0,78 & 0,74 & 0,83 & 0,75 & 0,84 & 0,72 & 0,66 & 0,53 & 0,64 & 0,62 \\
\hline $\mathrm{C}_{60}$ & - & 0,83 & 0,73 & 0,74 & 0,72 & 0,72 & 0,65 & 0,71 & 0,66 & 0,74 & 0,75 & 0,50 & $0,34 *$ & $0,44^{*}$ & $0,32 *$ \\
\hline $\mathrm{CAB}_{60}$ & - & - & - & 0,72 & 0,69 & 0,68 & 0,65 & 0,71 & 0,62 & 0,76 & 0,67 & 0,59 & 0,53 & 0,55 & 0,58 \\
\hline $\mathrm{P}_{120}$ & - & - & - & - & 0,97 & 0,91 & 0,89 & 0,97 & 0,93 & 0,88 & 0,89 & 0,83 & 0,63 & 0,81 & 0,71 \\
\hline $\mathrm{C}_{120}$ & - & - & - & - & - & 0,90 & 0,88 & 0,96 & 0,95 & 0,87 & 0,90 & 0,82 & 0,67 & 0,82 & 0,73 \\
\hline $\mathrm{CAB}_{120}$ & - & - & - & - & - & - & - & 0,82 & 0,81 & 0,81 & 0,78 & 0,79 & 0,66 & 0,79 & 0,74 \\
\hline $\mathrm{P}_{180}$ & - & - & - & - & - & - & - & - & 0,96 & 0,92 & 0,89 & 0,88 & 0,69 & 0,86 & 0,77 \\
\hline $\mathrm{C}_{180}$ & - & - & - & - & - & - & - & - & - & 0,86 & 0,85 & 0,83 & 0,71 & 0,82 & 0,74 \\
\hline $\mathrm{A}_{180}$ & - & - & - & - & - & - & - & - & - & - & 0,85 & 0,82 & 0,69 & 0,81 & 0,75 \\
\hline $\mathrm{CAB}_{180}$ & - & - & - & - & - & - & - & - & - & - & - & 0,81 & 0,61 & 0,71 & 0,64 \\
\hline $\mathrm{P}_{240}$ & - & - & - & - & - & - & - & - & - & - & - & - & 0,79 & 0,94 & 0,89 \\
\hline $\mathrm{C}_{240}$ & - & - & - & - & - & - & - & - & - & - & - & - & - & 0,81 & 0,82 \\
\hline $\mathrm{A}_{240}$ & - & - & - & - & - & - & - & - & - & - & - & - & - & - & 0,92 \\
\hline
\end{tabular}

(*) $\mathrm{P}<0,05$

( ) $\mathrm{P}<0,01$

A correlação entre o peso e o comprimento total, na mesma idade, apresentou valores altos e significativos $(\mathrm{P}<0,01)$, o que esta de acordo com Tave e Smitherman, (1980) aos 45 e 90 dias de idade, na tilápia (Oreochromis niloticus); Busack ; Gall (1983) aos 60 dias de idade no mosquito fish (Gambusia affinis); El Ibiary et al. (1978) aos 84 e 158 dias de idade no catfish (Ictalurus punctatus); Bailei e Lodenslager (1986) aos 84 e 180 dias de idade no salmão (Salmo salar); Reagan, Pardue e Eisen (1976) aos 150 dias de idade no Catfish; Refstie e Steine (1978) na idade de 210 dias no salmão; Klupp (1979) na idade de 270 dias na truta (Salmo gairdneri) e Rocha (1995) nas idades de 60, 90, 150, 210, 270 e 330 dias no curimbatá (Prochilodus lineatus).

As correlações entre altura e peso; altura e comprimento total; altura e comprimento da cabeça; comprimento da cabeça e peso; comprimento da cabeça e comprimento total apresentaram valores altos e significativo $(\mathrm{P}<0,01)$, e estão de acordo com valores encontrados por Rocha (1995), em idades entre 60 a 330 dias no curimbatá. As correlações entre as características peso em diversas idades, apresentaram valores de moderado a alto $(\mathrm{P}<0,01)$, o que coincide com os valores encontrados por El-Ibiary et al (1978) no catfish.

As correlações entre a característica comprimento corporal nas diversas idades apresentaram valores de moderado a alto $(\mathrm{P}<0,01)$ e valor baixo de 0,34 $(\mathrm{P}<0,05)$. Os valores moderado a alto concordam com Aulstad, Gjedrem e Skjervold (1972) e Silverstein, Hershberger (1995).

As correlações entre as características nas diversas idades apresentaram valores de moderado a alto $(\mathrm{P}<0,01)$, à exceção do comprimento total na idade de 60 dias com o peso na idade de 240 dias $(0,34)$ $(\mathrm{P}<0,05)$, comprimento total na idade de 60 dias e altura na idade de 240 dias $(0,44)(\mathrm{P}<0,05)$ e comprimento total na idade de 60 dias com comprimento da cabeça na idade de 240 dias $(0,32)(\mathrm{P}<0,05)$.

\section{Conclusões}

As fêmeas apresentaram valores médios para as características maiores do que os machos. No entanto, estas diferenças não foram estatísticamente significativas. 
O crescimento alométrico negativo foi observado na maioria das relações, a exceção de peso corporal e altura corporal, peso corporal e comprimento da cabeça.

Os valores de correlação entre as características nas diversas idades apresentaram valores moderados a altos para a maioria das associações, à exceção do comprimento corporal (60 dias) com algumas características na idade de 240 dias, que apresentaram valores baixos.

\section{Referências}

AULSTAD, D.; GJEDREM, T.; SKJERVOLD, H. Genetic and environmental sources of variation in length and weight of rainbow trout (Salmo gairdneri). J. Fish Res. Board. Can., Ottawa, v.29, p.237-241, 1972.

BAILEI, J.K.; LOUDENSLAGER, E.J. Genetic and environmental components of variation for growth of juvenile atlantic salmon (Salmo salar). Aquaculture: An International Journal Devoted To Fundamental Aquatic Food Resources, Amsterdam, v.57, p.125-132, 1986.

BUSACK, C.A.; GALL, G.A.E. An initial description of the quantitative genetics of growth and reproduction in the mosquito fish (Gambusia affinis). Aquaculture: an International Journal Devoted to Fundamental Aquatic Food Resources, Amsterdam, v.32, p.123-140, 1983.

EL-IBIARY, H.M. et al. Phenotypic correlations between commercial characters in channel catfish. Proc. Ann. Conf. Southeast. Assoc. Fish. Wild. Agen., v.32,p.420-425, 1978.

EUCLYDES,R.F. Manual de utilização do programa SAEG (Sistema para análise estatística e genética). Viçosa: Imprensa Universitária, 1983. 59p.

GJEDREM, T. Genetic variation in quantitative traits and selective breeding in fish and shellfish. Aquaculture: an International Journal Devoted to Fundamental Aquatic Food Resources, Amsterdam, v. 33, p.51-72, 1983.

GJERDE, B.; SCHAEFFER, L.R. Body traits in rainbow trout.II- Estimates of heritabilities and of phenotypic and genetic correlations. Aquaculture: An International Journal Devoted To Fundamental Aquatic Food Resources, Amsterdam, v. 80, p. 25-44, 1989.

GUNNES, K.; GJEDREM, T. A genetic analysis of body weigth and length in rainbow trout reared in seawater for
18 months. Aquaculture: an International Journal Devoted to Fundamental Aquatic Food Resources, Amsterdam, v.24, p.161-174, 1981.

KLUPP, R. Genetic variance for growth in rainbow trout (Salmo gairdneri). Aquaculture: an International Journal Devoted to Fundamental Aquatic Food Resources, Amsterdam, v.18, p.123-134, 1979.

LE CREN, E.D. The lenght-weight relationship and seasonal cycle in gonad weigth and condition in the perch (Perca fluviatis). Journal Animal Ecology, Oxford, v.20, p.201-219, 1951.

PADUA,H.B. Teores recomendados para manutenção de organismos aquáticos de água doce. Panorama da Aquícultura, Rio de Janeiro, v.4, n.21, p.8-9, 1994.

REAGAN, R.E.; PARDUE, G.B.; EISEN, E.J. Predicting selection response for growth of channel cat fisk. Journal Heredity, Washington, v.67, p.49-53, 1976.

REFSTIE, T.; STEINE, T.A. Selection experiments with salmon. III- Genetic and environmental sources of variation in length and weigth of atlhantic salmon in the fresh water phase. Aquaculture: an International Journal Devoted to Fundamental Aquatic Food Resources, Amsterdam, v.14, p. 221-234, 1978.

RICKER, W. E. Growth rates and models. In: HOAR, W.S; RANDALL D.J; BRETT, J.R. (Eds). Fish physiology bioenergetics and growth. New York : Academic Press, 1979. 786p.

ROCHA, M.A. Estimativas de herdabilidade e correlações genéticas,fenotípicas e ambientais de características avaliadas no curimbatá (Prochilodus lineatus), em idades entre 60 a 330 dias. 1995. 150 f. Dissertação (Doutorado em Aquícultura) - Centro de Aquícultura, UNESP, Jaboticabal, 1995.

ROYCE, W.F. Introduction to the fishery science. New York: Academic Press. 1972.

SILVERSTEIN, J.T.; HERSHBERGER, W.K. Genetics of size and growth rate through sexual maturity in freshwater-reared coho salmon (Oncorhynchus kisutch). Theorical and Applied Genetics, Berlin, v.90, n.5, p.733$739,1995$.

SOKAL, R.R.; ROHLF, F.J. Introduccion a la bioestadistica. Barcelona : Editorial Revert., 1980.

TAVE, D. ; SMITHERMAN, R.O. Predicted response to selection for early growth in tilapia nilotica. Trans. Am. Fish. Soc., v.109, p.439-445, 1980. 